\title{
Gamificación, aprendizaje divertido: propuesta pedagógica en la universidad península de Santa Elena
}

Alfredo Ramón Tumbaco Reyes
atumbaco@ upse.edu.ec
ORCID: 0000-0001-6469-7191

Docente de La Universidad Estatal Peninsula de Santa Elena

Esther Teresa Roca Quirumbay ster14_21@hotmail.com ORCID: 0000-0003-3715-3054

Docente de La Unidad Educativa José Pedro Varela

César Oswaldo Roca Quirumbay roqua222@hotmail.com ORCID: 0000-0002-5522-5325

Docente de La Unidad Educativa Dr. Luis Celleri Aviles

Tanya María Villón Gonzalez villontanya@gmail.com ORCID: 0000-0003-4512-2645

\section{RESUMEN}

Docente de La Unidad Educativa José Pedro Varela

La gamificación cada vez gana terreno en la educación superior y en otros contextos educativos en sus distintos niveles pedagógicos ante la actual coyuntura es una propuesta para los estudiantes del Preuniversitario y primer año en la Universidad Estatal Península de Santa Elena, como una estrategia de aprendizaje lúdica, el enfoque de investigación es una metodología interactiva, la participación de los estudiante se los analizó de forma cualitativa, los resultados se los midió mediante un test en gianelly.

La propuesta de la gamificación es un estilo de aprendizaje útil que potencia la motivación y la concentración del estudiante, la búsqueda de la información se las realizó a través de Google académico, artículos de revistas indexadas, libros y periódicos confidenciales, para conocer cuál es el rol principal que han tenido la gamificación y el juego en tiempo de pandemia, y el impacto que ha ocasionado.

La gamificación surge por la necesidad que demanda la educación actual y que se basan en el principio de la educación permanente a través de la tecnología digital como un derecho a la educación, la gamificación basado en juegos, se han convertido en una estrategia de enseñanza aprendizaje en tiempo de confinamiento convirtiéndolos en una oportunidad.

Palabras clave: educación superior; gamificación; juegos; gianelly; test 


\title{
Gamification, fun learning: pedagogical proposal at the universidad peninsula de Santa Elena
}

\begin{abstract}
Gamification is increasingly gaining ground in higher education and in other educational contexts at its different pedagogical levels in the current situation, it is a proposal for preuniversity and first-year students at the Santa Elena Peninsula State University, as a playful learning strategy., the research approach is an interactive methodology, the participation of the students was analyzed in a qualitative way, the results were measured by means of a test in gianelly.

The proposal of gamification is a useful learning style that enhances the motivation and concentration of the student, the search for information was carried out through academic Google, articles from indexed magazines, books and confidential newspapers, to know what the main role that gamification and gaming have had in times of pandemic, and the impact it has caused.

Gamification arises from the need that the current demands and that is based on the principle of permanent education through digital technology as a right to education, gamebased gamification has become a teaching-learning strategy in time of confinement turning them into an opportunity.
\end{abstract}

Key words: higher education; gamification; games; gianelly; quiz.

Artículo recibido: 30 noviembre. 2021 Aceptado para publicación: 29 diciembre 2021

Correspondencia: atumbaco@upse.edu.ec Conflictos de Interés: Ninguna que declarar 


\section{INTRODUCCIÓN}

La gamificación desde el punto de vista académico cada vez gana terreno en la educación superior y en otros contextos educativos quienes deben innovar en sus distintos niveles pedagógicos, y la comunidad debe ser parte de aquello, la gamificación es un enfoque de aprendizaje que traslada la mecánica de los juegos al ambiente formativo en el aula universitaria, con el objetivo de obtener mejores resultados, mejorar algunas habilidades y destrezas en los alumnos, o bien recompensar acciones concretas. En cuanto a la calidad de enseñanza y aprendizaje del docente universitario han tenido que valorizar seriamente su rol de educador para dar un cambio significativo a su estructura pedagógica dejar lo tradicional para innovador con la tecnología, es por eso, que es importante evocar que es la establecimiento universitario la que se anexa en el procesos de formación profesional académico, en donde la educación superior es un elemento necesario en el bienestar de los estudiantes.

Ante las dificultades mencionadas florece una nueva tendencia en el contexto educativo universitario, la innovación, donde se hace necesario implementar un nuevo enfoque metodológico denominado gamificación. La gamificación facilita la interiorización de conocimientos empleando un aprendizaje lúdico en un entorno divertido por media una herramienta "Gianelly". Como una propuesta que funciona y que gana terreno entre los profesores como una respuesta motivadora y efectiva para los estudiantes en las diferentes disciplina del currículo universitario.

La tecnología generar vínculos entre profesores y alumnos, y que actúan como ventana al mundo, se da en el caso de las cMOOCs (MOOC más colaborativos), las wikis, los Entornos Personales de Aprendizaje (PLE), la Pizarra Digital Interactiva (PDI), el mobile learning, y, cómo no, las técnicas de gamificación (Peñalva et al, 2019, p.247)

\subsection{Historia de la gamificación}

La gamificación siempre ha estado presente en la educación es por ello que cuando a los niños cuando le damos una crema de zanahoria, jugamos con la cuchara y la sopa le hacemos como si fuese un avión y si se la toma les decimos bravo, reconocimiento, refuerzo positivo y de pronto le dibujamos una carita feliz, eso es gamificación se lo ha hecho siempre, el primer juego de gamificación de la historia "El juego Real de Ur 2600 A.C" se lo exhibe en el Museo Británico (Londres), descifradas como juegos de mesa, 
este juego se lo realiza dos jugadores, cada uno con siete fichas (negras y blancas, como el tablero del juego de las damas quizás el ajedrez), y se usaban tres dados tetraédricos, un ejemplo de juego más moderno en este escenario contemporáneo para tomar en cuenta a nuestras clases son Boy Scauts creado a principio del siglo XX, un sistema gamificados en valores, se trata de un esquema de juegos destinados a aprender y poner en práctica las numerosas habilidades que los niños y adolescente deben conocer. Con las maquinas juegos por medio de computadoras un estudio sobre juegos el británico Nick Pelling, "señala que en el año 2002 inicia en el desarrollo de su trabajo, en el mundo de las computadoras, una vinculación de elementos propios del videojuego en el mundo de la programación informática" (Olivat, 2016, p.30).

En la era digital la gamificación es impulsada a través de entornos virtuales más sofisticada donde los estudiantes podrán aprender de una forma natural y lúdica., para este estudio de gamificación se va a utilizar una aplicación tecnológica genially que trabaja con elementos gamificados propios de su juego en cualquier escenario educativo para que estudiantes se divierta mientras aprende. Según Peña (2021), considera que software Gianelly "ofrece gran cantidad de recursos para aplicar la gamificación, una estrategia para potenciar el aprendizaje y la enseñanza”, de acuerdo al autor la herramienta tecnológica muestra una nueva dimensión en la creación de experiencias visuales, imágenes con interactividad y animación de forma muy sencilla, sin necesidad de saber programación, diseño una web o diseño gráfico, simplemente creando una cuenta gratuita o ingresando con tu correo de Gmail, aplicas una plantilla y empieza a diseñar tu juego interactivo. Según González (2019), "la gamificación en el aula es una estrategia de innovación educativa que en los últimos años ha sido adoptada por miles de docentes innovadores que desean aumentar la motivación por el aprendizaje en su alumnado" (p.1).

La gamificación y las herramientas tecnología siempre van a estar ligadas de forma indiscutible porque están a la vista de todos, prometen numerosas posibilidades de estrategias muy asombrosas para conectar usuarios y compartir recursos.

\subsection{Estudios realizados sobre gamificación}

Según García (2019), sostiene que la gamificación obtuvo una relación positiva de 0.249 $\mathrm{y}$ un valor de significancia de $\mathrm{p}=0.017<0.05$, concluyendo la existencia de una relación directa, determinando que cuanto mayor es el empleo de la gamificación existe un mayor 
desarrollo de las competencias matemáticas, por otra parte, autores como IIllescas et ál. 2020, consideran que los educadores, al usar la gamificación mejoran el aprendizaje de las matemáticas y despierta la motivación en los estudiantes, sin embargo, no la aplican con frecuencia de manera inadecuada; esto quiere decir que existe cierto grado de desconocimiento de la metodología, por lo tanto los resultados no son los esperados, otros autores también consideran que el uso de la gamificación con una herramientas tics tiene sus desventajas porque muchos docentes les hace falta capacitación, además la institución no cuenta con una buenas estructura tecnológica, además, no todos los estudiantes cuenta con un dispositivo electrónico, y un internet de banda ancha, según Banco Interamericano de Desarrollo (2019), todos estos inconvenientes reduce la motivación intrínseca, aumentos de la ansiedad y efectos motivacionales de corta duración.

Rodríguez et al. (2019), Establecen que "La gamificación puede ser una herramienta de aprendizaje universal, lo que evidencian las múltiples incorporaciones de juegos en la enseñanza", La gamificación fomenta la participación, la creatividad y la curiosidad, motiva a los alumnos por aprender porque están interactuando de manera directa en un entorno natural que le genera mucha confianza, es un escenario propicio para el aprendizaje.

El Banco Interamericano de Desarrollo (2019), considera que los estudios sobre gamificación han aumentado significativamente. Según Google Scholar, el número de artículos publicados anualmente que contienen las palabras "gamification" y "education" pasó de 140 en 2010 a 3.570 en 2014 y llegó a 9.570 en 2018 .

El Instituto para el Futuro de la Educación (2019), llevo a cabo un proyecto educativo relacionado con gamificación clarifica los mitos y realidades alrededor del tema, esta conversación se unieron 150 profesores de varias partes del mundo con el mismo interés de saber cómo aplicar gamificación para un mejor aprovechamiento del aprendizaje. En el webinar se planteó la siguiente interrogante: ¿Has implementado gamificación? El 47\% de los consultados dijeron que planea hacerlo, un $32 \%$ manifestaron que si fue una experiencia educativa positiva, y un $16 \%$, simplemente dijeron que no, y finalmente el $5 \%$, contestaron, Si pero no obtuve los resultados que esperaba.

\section{ESTRATEGIAS METODOLÓGICAS O MATERIALES Y MÉTODOS}

\subsection{En el contexto de caso la gamificación como pilar de eficiencia y eficacia en el rendimiento educativo universitario}


La gamificación es una estrategia metodológica que tiene firme propósitos dentro del contenido curricular universitario, donde el eje principal es recompensar o premiar el esfuerzo del alumno que está atento a una clase o que se esmera en cumplir de forma fiel los indicadores de logros; además para aquellos que no logran el objetivo también penaliza a su poco interés en aprender. La gamificación es un fenómeno que cada vez está más presente en el aula, específicamente con la tecnología y todas sus aplicaciones en el campo educativo. Dicha herramienta consiste en utilizar las mecánicas del juego con el fin de potenciar la motivación, la concentración o el esfuerzo, creando una mejora del proceso de enseñanza aprendizaje (García et ál. 2017).

Este estudio se centra en el contexto de la Universidad UPSE en estudiante del preuniversitario de la asignatura Fundamentos de Programación, entidad pública perteneciente a la provincia de Santa Elena, su comunidad educativa y sus estudiantes tienen diferentes niveles socio cultural y económico, el estudio se desea demostrar que gamificación es una estrategia didáctica para potenciar el aprendizaje y la enseñanza, para eliminar el aburrimiento y la escasez de motivación en las clases de programación, ponerla en marcha esta propuesta servirá de apoyo para los profesores y ayudará a los alumnos a desarrollar esas habilidades y destrezas que todavía no han sido explotadas. A continuación, la propuesta: Diseñar una propuesta gamificada para conseguir los objetivos por medio de la de la herramienta genially, para potenciar la enseñanza y el aprendizaje aplicando test de preguntas, el objetivo principal de este estudio es generar contenidos interactivos lúdicos que ayuden a potenciar el aprendizaje en la asignatura de programación. Asimismo se planifico otros objetivos secundarios para cumplir con la meta: Ofrecer variedad de recursos para aplicar la gamificación, como estrategia para potenciar el aprendizaje y la enseñanza; Crear test interactivos que permita desarrollar feedback en tiempo real, mejorando las habilidades del estudiante; Diseñar infografía, presentaciones y quiz para facilitar la asimilación de conceptos complejos.

\subsection{Metodología:}

\subsubsection{Descripción de las actividades y/o actuaciones ideadas para innovar y/o mejorar la docencia}

En este estudio se adopta a un enfoque cuantitativo, basado en un estudio práctico, en la Universidad Península de Santa Elena UPSE, con grupo de participantes inscritos de 40 estudiantes divido en dos grupos A y B que cursan el preuniversitario, en un diagnóstico 
previo que se ejecutó en la apertura del año lectivo sobre conocimiento general de la asignatura de programación sobre comprensión de los contenidos básico, los estudiantes manifestaron en la prueba que esos contenidos eran complicados y difícil de comprender, que no lo habían repaso en la etapa anterior colegial, asilo lo determinó en un análisis el docentes que imparte la asignatura, quien pudo constatar en una evaluación previa evaluada de forma tradicional sin la tecnología mediante un papel con preguntas largas difícil de completar y entender, esta situación negativa hizo que haya gran desmotivación por aprender la programación, los estudiantes obtuvieron una calificación por debajo de 7, que hasta ese momento sería un supletorio, la problemática se lo asocia a algunas razones en algunos colegio no se imparte la programación como una asignatura base de un pensum, otra razón la desmotivación por no comprender la metodología de enseñanza en la asignatura programación al obtener una nota mínima, y por otra parte la desmotivación dado por el desarrollo del nivel de dificultad de la asignatura.

Para Escudero et al, (2008), Un estudio de caso es "Una metodología de análisis y observación de situaciones específicas con mucha profundidad, como por ejemplo, biografías de personajes, historia de instituciones", para Martínez (1998), el estudio de caso "Esla búsqueda e interpretación de los datos y fenómenos requerido en tales estudio, la mayoría se los realizaba por el métodos descriptivos.

La propuesta se desarrolla en base a los de los contenidos curriculares en temas difíciles de la programación y se los orientados a la gamificación, para fomentar la participación y la motivación de los jóvenes para que se logre mejorar la comprensión de los contenidos y la práctica de como programar.

Este estudio se basa en una metodología de investigación interactiva lúdica por medio de la gamificación usando la herramienta Genially, aplicando QUIZ, se trabajó con dos grupos de estudiantes del preuniversitario y de primer año de la asignatura programación un total 40 alumnos que oscilan entre 18 a 22 años, todos universitarios cuenta con un dispositivo electrónico con capacidades básicas de conexión para dar respuesta a las actividades propuestas en la gamificación.

El método de enseñanza aplicados en estos contenidos curriculares, es el aprendizaje basado en juego donde se aplicó los recurso de la plataforma gianelly quien crea una gamificación para triunfar tan solo usando plantillas basadas en juegos con dinámicas divertidas, este software aprovecha lo mejor de las dinámicas habituales del juego para 
potenciar el aprendizaje, la enseñanza y la formación lo que hay que hacer es plantear una asignatura en este caso programación con mecánicas de juego con puntos y niveles, y por supuesto que los alumnos prestan más atención y se involucran más en el aprendizaje. Los tópicos que se abordan en la planificación de la propuesta fueron extraídos del silabo de la malla del preuniversitario.

La propuesta cuenta con dos temas que pasaran a ser dos sesiones, cada una se enlaza con el objetivo del estudio, cada sesión se ejecuta activando los conocimientos previos que tienen los alumnos, con el propósito de crear los nuevos conocimientos. A continuación, se realizan algunas actividades en la plataforma Gianelly en un formato gamificados como estrategia para lograr el aprendizaje de forma activa, la evaluación se la realizó por medio de los test, quiz-pizarra-magnética-programación, quiz-pizarra-magnéticaprogramación-preuni, trabajos individuales, al concluir las actividades se estimula al alumno otorgándoles distintivos, como puntos, insignia, premios, recompensa, etc.

Al finalizar el estudio, se creó un test de satisfacción en google forms, se los aplicó a los estudiantes de los grupo A y B, para lograr una evaluación de los temarios.

\subsubsection{Tiempo de ejecución de las sesiones de trabajo}

La ejecución del estudio se lo estableció en dos semanas de 40 minutos, aplicando la flexibilidad en el proceso de ejecución tal como lo recomienda la Ley de educación superior y los principios de la Constitución de la Republica del Ecuador.

\section{Tabla 1}

Sesión Uno Quiz-Pizarra-Magnética-Programación-Preuni

\begin{tabular}{llc}
\hline \multicolumn{1}{c}{ Sesión uno } & \multicolumn{1}{c}{ Tipo de espacio: Virtual } & Tiempo: \\
\hline $\begin{array}{l}\text { Objetivos de } \\
\text { la sesión }\end{array}$ & $\begin{array}{l}\text { Conocer la historia de los enfoques de la programación, } \\
\text { realizando los ejercicios prácticos en él, quiz-pizarra- } \\
\text { magnética-programación-preuni }\end{array}$ & $\begin{array}{c}40 \\
\text { minutos }\end{array}$ \\
\hline \multirow{5}{*}{ Actividades } & $\begin{array}{l}\text { Analizar y responder los retos creados en la herramienta geneally } \\
\text { https://view.genial.ly/61e4d3e45c54fb0cd8963f53/interactive-content- } \\
\text { quiz-pizarra-magnetica-programacion-preuni, Cada reto ejecutado } \\
\text { correctamente tiene el valor de 1 puntos cada una. }\end{array}$ \\
\hline Recursos & $\begin{array}{l}\text { Internet, Herramienta Gianelly, C++, Herramienta WhatsApp, Zoom, } \\
\text { Computadora, Celular }\end{array}$ \\
\hline Evaluación & $\begin{array}{l}\text { Se evaluará mediante la herramienta Gianelly test interactivo de 10 } \\
\text { preguntas de opciones múltiple cada pregunta tiene el valor de 1 punto. }\end{array}$ \\
\hline
\end{tabular}

Fuente: Elaboración propia 


\section{Tabla 2}

Sesión Dos, Quiz-Pizarra-Magnética-Programación

\begin{tabular}{|c|c|}
\hline Sesión dos & Tipo de espacio: Virtual \\
\hline Objetivos de & Evaluar la unidad de trabajo de la asignatura de programación por \\
\hline la sesión & medio de la gamificación, quiz-pizarra-magnética-programación \\
\hline Contenido & Tipos de variables \\
\hline \multirow{4}{*}{ Actividades } & Ejecutar un reto creado en la herramienta geneally. \\
\hline & https://view.genial.1y/61e454ae55825d0ce86837b9/interactive- \\
\hline & content-quiz-pizarra-magnetica-programacion, Cada reto ejecutado \\
\hline & correctamente tiene el valor de 1 puntos cada una. \\
\hline \multirow{2}{*}{ Recursos } & Internet, Herramienta Gianelly, C++, Herramienta WhatsApp, Zoom, \\
\hline & Computadora, Celular \\
\hline Evaluación & Se evaluará mediante la herramienta Gianelly test interactivo de 10 \\
\hline & preguntas de opciones múltiple cada pregunta tiene el valor de 1 punto. \\
\hline
\end{tabular}

Fuente: Elaboración propia

\subsubsection{Cronograma}

El orden de la propuesta temporal de las actividades gamificadas se ha llevado a cabo por fechas establecidas, desarrollado en sesiones de 60 minutos, y estará al mando del profesor de aula, con el propósito de mejorar la motivación de los alumnos por medio de la nueva propuesta pedagógica.

\section{Tabla 3}

Sesiones Por Semanas Test Realizado En Agosto De 2021

\begin{tabular}{|c|c|c|c|c|c|c|c|c|c|c|}
\hline & \multicolumn{5}{|c|}{ Semana1 } & \multicolumn{5}{|c|}{ Semana2 } \\
\hline & $\mathbf{L}$ & $\mathbf{M}$ & $\mathbf{M}$ & $\mathbf{J}$ & V & $\mathbf{L}$ & $\mathbf{M}$ & $\mathbf{M}$ & $\mathbf{J}$ & $\mathbf{V}$ \\
\hline Sesión 1 & $\mathbf{x}$ & & & & & & & & & \\
\hline Sesión 2 & & & & & & & & $\mathrm{x}$ & & \\
\hline
\end{tabular}

Fuente: Elaboración propia

\section{RESULTADOS Y DISCUSIÓN}

Se trabajó con dos actividades gamificadas en la plataforma gianelly para cada grupos de estudiante matriculados en el preuniversitario y primer año, se socializó las instrucciones en clase cómo funcionaban los quiz, las preguntas, fueron propicias a los temas distribuida en el paralelo en las dos sesiones, los test gamificados se encuentran en el siguiente en la 
siguiente plataforma ver enlace https://genial.ly/es/, puede ingresar con algún correo o suscribiéndose a la plataforma.

Cabe destacar que el trabajo se realizó sin contratiempos, sin inconvenientes, todos los estudiantes lograron responder las preguntas de los test. Asimismo, los estudiantes se enlazaron sin problemas desde sus domicilios y respondieron los quiz al ritmo establecido por el docente universitario.

Minutos después de haber ejecutado la práctica de los test, los universitarios capturaron sus respuestas y lo enviaron al profesor por medio del programa de mensajería instantánea WhatsApp, con esas muestras el investigador diseño una tabla de resultados. De esta manera el pedagogo pudo cruzar las estadísticas de cada pregunta, más el puntaje final de cada universitario en cada test, en la tabla se agregó el total de respuestas correctas e incorrectas. Se diseñó una tabla de calificación del 1-10, según como estaba establecida la puntuación y la cantidad de respuestas correctas obtenidas por el alumno, en este proceso se otorgó la calificación máxima de (10). La evidencia de las calificaciones de los A $\quad$ y $\quad$ B se encuentra en el siguiente enlace: https://docs.google.com/spreadsheets/d/1HLTiaqmmESOiEY-

\section{U3KxYKmddlFtirB5/edit\#gid=1367074597.}

La evidencia de las preguntas aplicadas a los estudiantes de los dos grupos A y B está en el drive, se encuentra en este link, los temarios fueron revisados por el área de informática. https://drive.google.com/drive/folders/1KaIB6SAIk_W5EhKC_-iWY27eHoJWYiyc

En las siguientes tablas se muestran los aciertos y desaciertos, las calificaciones finales y el promedio del curso.

\section{Tabla 4}

Resultados De Acierto Y Calificaciones Del Primer Grupo.

\begin{tabular}{|c|c|c|c|c|c|c|c|c|c|c|c|c|c|c|}
\hline \multirow{2}{*}{ Grupo A } & \multicolumn{11}{|c|}{ Aciertos } & \multirow{2}{*}{ P.Correctas } & \multirow[b]{2}{*}{ P.Incorrectas } & \multirow{2}{*}{ Calificación } \\
\hline & 0 & 1 & 2 & 3 & 4 & 5 & 6 & 7 & 8 & 9 & 10 & & & \\
\hline $\mathrm{A} 1$ & & & & & & & & $\mathrm{x}$ & & & & 7 & 3 & 7 \\
\hline $\mathrm{A} 2$ & & & & & & & & & & & $\mathrm{x}$ & 10 & 0 & 10 \\
\hline A3 & & & & & & & & & & $\mathrm{x}$ & & 9 & 1 & 9 \\
\hline A4 & & & & & & & & & & & $\mathrm{x}$ & 10 & 0 & 10 \\
\hline A5 & & & & & & & & & & & $\mathrm{x}$ & 10 & 0 & 10 \\
\hline A6 & & & & & & & & & & $\mathrm{x}$ & & 9 & 1 & 8 \\
\hline A7 & & & & & & & & & & $\mathrm{x}$ & & 9 & 1 & 9 \\
\hline A8 & & & & & & & & & & & $\mathrm{x}$ & 10 & 0 & 10 \\
\hline A9 & & & & & & & & & & $\mathrm{x}$ & & 9 & 1 & 9 \\
\hline
\end{tabular}




\begin{tabular}{|c|c|c|c|c|c|c|c|c|c|c|c|c|c|}
\hline \multirow{2}{*}{ Grupo A } & \multicolumn{10}{|c|}{ Aciertos } & \multirow{2}{*}{ P.Correctas } & \multirow{2}{*}{ P.Incorrectas } & \multirow{2}{*}{ Calificación } \\
\hline & 0 & 1 & 2 & 3 & $4 \quad 5$ & 6 & 7 & 8 & 9 & 10 & & & \\
\hline A10 & & & & & & & & & $\mathrm{x}$ & & 9 & 1 & 9 \\
\hline A11 & & & & & & & & & $\mathrm{x}$ & & 9 & 1 & 9 \\
\hline A12 & & & & & & & & & & $\mathrm{x}$ & 10 & 0 & 10 \\
\hline A13 & & & & & & & & & $\mathrm{x}$ & & 9 & 1 & 9 \\
\hline A14 & & & & & & & & & & $\mathrm{x}$ & 10 & 0 & 10 \\
\hline A 15 & & & & & & & & & & $\mathrm{x}$ & 10 & 0 & 10 \\
\hline A16 & & & & & & & & & & $\mathrm{x}$ & 10 & 1 & 10 \\
\hline A17 & & & & & & $\mathrm{x}$ & & & & & 6 & 4 & 6 \\
\hline A18 & & & & & & & & $\mathrm{x}$ & & & 7 & 3 & 8 \\
\hline A19 & & & & & & & & & & $\mathrm{x}$ & 10 & 0 & 10 \\
\hline A20 & & & & & & & & & $\mathrm{x}$ & & 9 & 1 & 9 \\
\hline Total & & & & & & & & & & & 182 & 19 & 9,1 \\
\hline
\end{tabular}

Fuente: Elaboración propia

\section{Tabla 5}

Resultados De Acierto Y Calificaciones Del Segundo Grupo

\begin{tabular}{|c|c|c|c|c|c|c|c|c|c|c|c|c|c|c|}
\hline \multirow{2}{*}{ Grupo B } & \multicolumn{11}{|c|}{ Aciertos } & \multirow{2}{*}{ P.correctas } & \multirow{2}{*}{ P.Incorrectas } & \multirow{2}{*}{ Calificación } \\
\hline & 0 & 1 & 2 & 3 & 4 & 5 & 6 & 7 & 8 & 9 & 10 & & & \\
\hline B1 & & & & & & & & & & & $\mathrm{x}$ & 10 & 0 & 10 \\
\hline $\mathrm{B} 2$ & & & & & & & & & & $\mathrm{x}$ & & 9 & 1 & 9 \\
\hline B3 & & & & & & & & & & $\mathrm{x}$ & & 9 & 1 & 9 \\
\hline B4 & & & & & & & & & & & $\mathrm{x}$ & 10 & 0 & 10 \\
\hline B5 & & & & & & & & & & & $\mathrm{x}$ & 10 & 0 & 10 \\
\hline B6 & & & & & & & & & & $\mathrm{x}$ & & 9 & 1 & 9 \\
\hline B7 & & & & & & & & $\mathrm{x}$ & & & & 7 & 3 & 7 \\
\hline B8 & & & & & & & & & & & $\mathrm{x}$ & 10 & 0 & 10 \\
\hline B9 & & & & & & & & & & $\mathrm{x}$ & & 9 & 1 & 9 \\
\hline B10 & & & & & & & & & & $\mathrm{x}$ & & 9 & 1 & 9 \\
\hline B11 & & & & & & & & & & $\mathrm{x}$ & & 9 & 1 & 9 \\
\hline B12 & & & & & & & & & & & $\mathrm{x}$ & 10 & 0 & 10 \\
\hline B13 & & & & & & & & & & $\mathrm{x}$ & & 9 & 2 & 9 \\
\hline B14 & & & & & & & & & & $\mathrm{x}$ & & 9 & 1 & 9 \\
\hline B 15 & & & & & & & $\mathrm{x}$ & & & & & 6 & 4 & 6 \\
\hline B16 & & & & & & & & & & & $\mathrm{x}$ & 10 & 1 & 10 \\
\hline B17 & & & & & & & & & & & $\mathrm{x}$ & 10 & 0 & 10 \\
\hline B18 & & & & & & & & $\mathrm{x}$ & & & & 7 & 3 & 7 \\
\hline B19 & & & & & & & & & & $\mathrm{x}$ & & 9 & 1 & 9 \\
\hline B20 & & & & & & & & & & $\mathrm{x}$ & & 9 & 1 & 9 \\
\hline Total & & & & & & & & & & & & 180 & 22 & 9 \\
\hline
\end{tabular}

Fuente: elaboración propia 
Tabla 6 Promedio Alcanzado Por Dos Grupos Evaluado Con Gamificación

\begin{tabular}{ccc}
\hline Grupo A & Grupo B & Promedio de los dos curso \\
\hline 9,10 & 9 & 9,05 \\
\hline
\end{tabular}

\section{Fuente: Elaboración propia}

En la tabla 7, se observa que las evaluaciones de los grupos A y B, en las dos sesiones realizadas obtuvieron una calificación con un promedio muy aceptable en los dos grupos es de 9.05, todos los estudiantes pudieron rendir las pruebas sin inconvenientes y las preguntas eran totalmente sencillas, fácil de responder, este tipo de ensayo permite hacer retroalimentación (feedback) de la clase por medio de un juego, los jóvenes aprender de la mejor manera la asignatura.

Finalmente, en la tabla siete se muestra una encuesta de satisfacción que trata de recoger información necesaria sobre estudio de Gamificación, y el uso de la plataforma gianelly por medio de la escala de Likert, escala lineal, con una puntuación de 1 a 5 , según las siguientes opciones: 1 Totalmente en desacuerdo; 2 En desacuerdo; 3 indiferente; 4 De acuerdo; 5 Totalmente de acuerdo, las pregunta fueron realizado en la plataforma Google Forms y almacenado en Google DRIVE. A continuación el siguiente enlace https://docs.google.com/forms/d/1FJBxxLCTfXJVzDEJEQeR-cV-C7VkivntDf-

\section{_W5sSXDs/edit\#responses}

Tabla 7 Resultados Del Cuestionario De Satisfacción

\begin{tabular}{lcc}
\hline \multicolumn{1}{c}{ Preguntas } & Media 1-5 & Des/estándar \\
\hline $\begin{array}{l}\text { Prefiero realizar una prueba en un software informático como } \\
\text { gianelly frente a un paradigma de test tradicional. }\end{array}$ & 3,69 & 0,69 \\
$\begin{array}{l}\text { Al participar del test he aprendido el contenido de la asignatura } \\
\text { de programación de forma activa }\end{array}$ & 3,71 & 0,65 \\
$\begin{array}{l}\text { Al participar del test he aprendido el contenido de la asignatura } \\
\text { de programación de forma activa }\end{array}$ & 3,95 & \\
$\begin{array}{l}\text { Me ha gusta contestar los quiz de preguntas del examen } \\
\text { He superado la nota inicial, es más práctica que una prueba }\end{array}$ & 3,92 & 0,92 \\
tradicional & & 1,11 \\
$\begin{array}{l}\text { El proceso de la prueba de los test ha sido muy complicado para } \\
\text { mí }\end{array}$ & 3,00 & 0,81 \\
$\begin{array}{l}\text { Creo que útil poder tener acceso a las prueba creados por mis } \\
\text { docentes al menos en programación }\end{array}$ & 4,18 & 1,03 \\
$\begin{array}{l}\text { He utilizado los quiz de gianelly creado por los docentes para } \\
\text { estudiar. }\end{array}$ & 3,82 & 1,07 \\
$\begin{array}{l}\text { Es la primera vez que realizo un a prueba con esta estrategia } \\
\text { lúdica }\end{array}$ & 3,85 & \\
\hline
\end{tabular}

Fuente: Elaboración propia 


\section{CONCLUSIÓN O CONSIDERACIONES FINALES}

\subsection{Consideraciones finales}

En cuanto a la propuesta de la gamificación se centró en los esquemas de los contenidos de la planificación curricular de la asignatura programación, los puntos más valiosos que se resumirían en las consideraciones finales quedarían de la siguiente manera.

\subsection{Ventajas de la gamificación}

La gamificación como propuesta didáctica, fomentó el desarrollo de procesos de la enseñanza haciendo más divertidas la asignatura muy significativa para el aprendizaje de los estudiantes de la Universidad Estatal Península de Santa Elena. De la misma manera, las actividades propuestas contribuyeron a un debate entre los docentes en su labor pedagógica, con una visión orientada a la innovación. Además de favorecer la adquisición de conocimientos, la atención y concentración en el aula, en el proceso académico mejora las relaciones sociales.

La gamificación es un elemento importante que introduce todo el potencial de los juego al ambiente educativo, para el desarrollo de la formación del estudiante en clase y permite que el aprendizaje sea divertido y natural. Propone una cadena de actividades interesantes, divertidas, que relajan o motivan y que, en ocasiones, se han visto limitadas. La implantación de la actividad gamificada es una metodología didáctica que mejora las destrezas y habilidades en la enseñanza de los estudiantes universitarios.

Las universidades fiscales y particulares deben arriesgar y apostar por la innovación académica, romper paradigmas que ocasionan perjuicio a la educación, hay que iniciar nuevos enfoques de enseñanza, tener en cuenta que muchos contenidos son monótonos, aburridos y tradicionales, además que no incluyen las necesidades de la coyuntura actual e intereses de los alumnos. En la nueva innovación pedagógica debe trabajar con la trilogía educativa de forma mancomunada, con la comunidad, docentes y autoridades. Sincerarse y reconocer que hay que tomar acciones concretas para mejorar el aprendizaje del alumno mediante estrategias de la gamificación que faciliten áreas dinámicas y atractivos. Las cualidades de los alumnos aumentan cuando todos los involucrados en la educación se empoderan sobre los inconvenientes y la educación de los jóvenes se respeta recíprocamente.

Se ha probado que la gamificación es una técnica de aprendizaje que efectivamente funciona porque logra motivar a los estudiantes, esta estrategia se debe usar en el contexto 
educativo como parte de su praxis académica. Por ello, los profesores deben reflexionar, cuestionarse cuál es su rol, y cuando puedan elegir una adecuada metodología, para, de esta manera, colaborar con la educación de los escolares, apoyándolos al desarrollo de destrezas que le sumen para enfrentar su entorno social, estableciendo en el establecimiento educativo una responsabilidad con la formación de los alumnos.

La implementación de la gamificación en los diferentes espacios educativos se hace efectivo cuando se incluyen entrenamientos didácticos reales, con el fin de transformar las prácticas estudiantiles que promuevan la participación y la motivación de los jóvenes como actores principales y se favorezca el desarrollo de destrezas necesarias para afinar los procesos de aprendizaje.

Las actividades gamificadas, establecidas de modo adecuado, se convierten en una táctica positiva que puede ser asociada de manera individual o grupal. La gamificación no discrimina ni excluye, al contrario, acepta al individuo, porque reconoce la potencialidad y el talento, materializado por medio del descubrimiento oportuno.

\subsection{Inconvenientes en el proceso de investigación}

Ciertos profesores observaron que al implantar la gamificación en sus clase los alumnos se distraían por el juego por lo tanto se perdía el tiempo, asimismo surgieron problemas de comportamiento, y atraso en cuanto al ritmo de las clases, porque algunos estudiantes no les gusta la enseñanza con juego como toda cosa hay una excepción, se sentían molestos, aburridos. Hay que partir de la base de que no todos los jóvenes tienen las cualidades definidas para trabajar bajo esas metodologías. Si esta circunstancia sucede, es necesario insistir en la importancia y la necesidad de practicar la ejecución de unas distribuciones grupales.

Por otra parte, otro de los inconvenientes es que los progenitores no se empoderan de las actividades gamificada de los jóvenes en casa y en el establecimiento.

\subsection{Líneas de investigación futuras}

La gamificación tiene un impacto positivo en el aprendizaje por su acción motivadora e interactiva, se reflexiona sobre las posibles líneas de investigación futuras. En primer lugar, la posibilidad de llevar a cabo una intervención sobre aprendizaje con juegos lúdicos más ambicioso, donde se integren todas las asignaturas. Segundo la vinculación directa y la necesidad de profundizar en trabajos afines con la preparación académica del profesor en estos aspectos educativos. 
Diseñar un sitio web con herramientas de gamificación efectiva para el aprendizaje virtual, que permitan evaluar de forma sistemática la participación del estudiante, de acuerdo a la implantación de una plataforma educativa. Sería muy interesante llevar a cabo las actividades de enseñanza y aprendizaje dentro de un mundo virtual en tercera dimensión.

\subsection{Conclusión}

Los resultados obtenidos sobre la propuesta de la actividades gamificadas pueden ser usadas en nuevos contextos, pero en un mismo nivel educativo por ejemplo en asignatura del ciclo básico como matemáticas, lenguaje y comunicación, historia y en otras de nivel superiores, este experimento recoge gran valor en tiempo difíciles, pandémico que está creando nuevas forma de concebir la educación y que cambien en todos los niveles educativos.

Exclusivamente la educación universitaria es un desafío poder atender a todo el estudiantado desde la educación virtual como mayor reto en las etapas educativas. Por ello, es necesario insistir que la gamificación puede ser un enfoque metodológico ventajoso para favorecer la educación virtual presencial, y para aumentar la participación e incrementar su motivación.

Otras de las ventajas es que el estudiantes de esta era tecnológicas, son considerados nativos dígales, poseen mejores destrezas que los docente en la manipulación y uso de las herramientas y aplicaciones, son experto en el manejo de las diferentes entornos educativo que ofrece la informática.

\section{REFERENCIAS BIBLIOGRAFICAS}

Araya, R., Arias Ortiz, E., Bottan, N., \& Cristia, J. (2019). ¿Funciona la gamificación en la educación? Documento de trabajo del BID, 1-48.

Escudero Macluf, J., Delfín Beltrán* y, L. A., \& Gutiérrez González, L. (2008). El estudio de caso como estrategia de investigación en las ciencias sociales. Ciencia Administrativa, 2-4.

Games, A. (2014). El juego inherente al ser humano. Director de Diseñode Microsoft.

García Collantes , D. E. (2019). Gamificación y competencias matemáticas en los estudiantes de 6to grado de la I. E. 2071 César Vallejo, Los Olivos 2019. Tesis, Universidad César Vallejo. Obtenido de https://hdl.handle.net/20.500.12692/41937 
García Fernández, J., Fernández Gavira, J., Sánchez Oliver, A. J., \& Grimaldi Puyana, M. (2017). Gamificación y aplicaciones móviles para emprender: una propuesta educativa en la enseñanza superior. International Journal of Educational Research and Innovation, 248-259.

Gewerc, A., \& Zapata Ros, M. (2019). Presentación del número especial de RED sobre competencia digital e inclusión social en niños y niñas de educación primaria. RED. Revista de Educacióna Distancia, 5-6. doi:http://dx.doi.org/10.6018/red/61/00

González González, C. (2019). Gamificación en el aula: ludificando espacios de enseñanzaaprendizaje presenciales y espacios virtuales. Researchgate. net, 1-22.

Harter, S. (1981). A scale of intrinsic versusextrinsic orientation in the clasroom (Vol. 3). New York, Estados Unidos: University of Demver.

IIllescas Cárdenas, R. C., GarcíaHerrera, D. G., Erazo-Álvarez, C. A., \& Erazo-Álvarez, J. C. (2020). Aprendizaje Basado en Juegos como estrategia de enseñanza de la Matemática. Revista Interdisciplinaria de Humanidades, Educación, Ciencia y Tecnología. doi:10.35381/cm.v6i1.345

Lowenhaupt, R., \& Hopkins, M. (2020). Considerations for school leaders serving US immigrant communities in the global pandemic. Journal of Professional Capital and Community, 5(374), 375-380. doi:https://doi.org/10.1108/JPCC-05-20200023

Martínez Bonafé, J. (1988). El estudio de caso en la investigación educativa. Investigación en la escuela(6), 1-10.

Observatorio de Innovación Educativa del Tecnológico de Monterrey . (2016). Gamificación. Observatorio de Innovación Educativa, 1-36.

Oliva, H. A. (2016). La gamificación como estrategia metodológica en el contexto educativo universitario. Realidad y Reflexión, 1-19.

Peñalva , S., Aguaded, I., \& Torres Toukoumidis , Á. (2019). La gamificación en la universidad española. Una perspectiva educomunicativa. Mediterranea de Educación. doi:doi.org/10.14198/MEDCOM2019.10.1.6

Rodríguez Oroz, D., Gómez Espina, R., Bravo Pérez , M., \& Truyol, M. E. (2019). Aprendizaje basado en un proyecto de gamificación: vinculando la educación universitaria con la divulgación de la geomorfología de Chile. Revista Eureka 
Tumbaco Reyes y otros

sobre Enseñanza y Divulgación de, 16(2).

doi:https://doi.org/10.25267/RevEurekaensendivulgcienc.2019.v16.i2.2202

Román, R. (2019). Gamificación: mitos y realidades. Instituto para el futuro de la Educación. Obtenido de https://observatorio.tec.mx/edu-news/gamificacionmitos-y-realidades 